\title{
Towards Reproducible Enzyme Modeling with Isothermal Titration Calorimetry
}

Felix Ott, Kersten S. Rabe, Christof M. Niemeyer, Gudrun Gygli*

*corresponding author: gudrun.gygli@kit.edu

All authors are located at: Institute for Biological Interfaces (IBG 1), Karlsruhe Institute of Technology (KIT), Hermann-von-Helmholtz-Platz 1, 76344 Eggenstein-Leopoldshafen, Germany

GG conceived, designed and performed experiments. KSR, CMN and GG developed the concept and led experimental work. FO produced and purified Gre2 $p$ and designed and performed experiments. All authors wrote and reviewed the manuscript.

Keywords: (S)-stereoselective ketoreductase, kinetic model, FAIR/F.A.I.R., reproducibility, quality control, dynamic light scattering, UV-Vis spectrophotometry 


\section{Abstract}

To apply enzymes in technical processes, a detailed understanding of the molecular mechanisms is required. Kinetic and thermodynamic parameters of enzyme catalysis are crucial to plan, model, and implement biocatalytic processes more efficiently. While the kinetic parameters, $K_{m}$ and $k_{\text {cat }}$, are often accessible by optical methods, the determination of thermodynamic parameters requires more sophisticated methods. Isothermal titration calorimetry (ITC) allows the label-free and highly sensitive analysis of kinetic and thermodynamic parameters of individual steps in the catalytic cycle of an enzyme reaction. However, since ITC is susceptible to interferences due to denaturation or agglomeration of the enzymes, the homogeneity of the enzyme sample (HES) must always be considered, and this can be accomplished by means of dynamic light scattering (DLS) analysis. We here report, for the first time, on the use of an ITC-dependent work flow to determine both the kinetic and the thermodynamic data for a cofactor-dependent enzyme. Using a standardized approach with implementation of sample quality control by DLS, we obtain high quality data suitable for advanced modeling of the enzyme reaction mechanism. Specifically, we investigated stereoselective reactions catalyzed by the NADPH-dependent alcohol dehydrogenase Gre2p under different reaction conditions. The results revealed that this enzyme operates with an ordered sequential mechanism and is affected by substrate or product inhibition depending on the reaction buffer. Data reproducibility is ensured by specifying standard operating procedures (SOPs), using programmed workflows for data analysis, and storing all data in a F.A.I.R. (findable, accessible, interoperable, and re-usable) repository (https://fairdomhub.org/investigations/464\#projects). Our work highlights the utility for combined binding and kinetic studies for such complex multi-substrate reactions. 


\section{Introduction}

Enzymes are widely used biocatalysts that have excellent chemo-, regio-, and enantioselectivity that exceeds those of many chemical catalysts. ${ }^{1-3} \mathrm{~A}$ profound understanding of the reaction mechanism of an enzyme and how it is influenced by process conditions is essential for the successful implementation of a biocatalytic process. ${ }^{4-6}$ To elucidate mechanistic principles, the kinetics of enzymes can often be conveniently studied using UV-Vis spectrophotometric or fluorescence-based assays in high-throughput formats, such as multi-well plates. However, this approach requires optically detectable cofactors, substrates, or products to track their consumption or production, respectively, and it may also be limited by the lower or upper detection limit of the analyte. Furthermore, this methodology is not suited for measuring thermodynamic binding parameters, although these can be crucial for understanding reaction mechanisms. To overcome these limitations, and to obtain in-depth insights into the biomolecular interactions and kinetic parameters of enzyme reactions, isothermal titration calorimetry (ITC) can be used. ITC is a highly sensitive, label-free analysis method that is being developed for more than two decades as a powerful tool to measure the thermodynamic parameters of biomolecular interactions (binding parameters, $K_{d}, \Delta \mathrm{H}_{\text {binding, }} \Delta \mathrm{G}^{\circ},-\mathrm{T} \Delta \mathrm{S}$ and the stoichiometry of binding), ${ }^{7,8}$ and has recently gained acceptance to measure parameters of enzyme kinetics (kinetic parameters, $K_{m}$ and $\left.k_{\text {cat }}\right){ }^{8-10}$

Three types of ITC experiments, traditional binding experiments (ITC-BIND), experiments using the multiple injection method (ITC-MIM), and experiments using the single injection method (ITC-SIM), are usually applied to measure the heat generated by binding interactions or by the enzymatic reaction itself. In the traditional binding experiment, a ligand is titrated to the enzyme. The resulting titration peaks are then integrated to calculate the enthalpy of binding $\left(\Delta \mathrm{H}_{\text {binding }}\right)$ and fit the dissociation constant $\left(K_{d}\right)$. From the enthalpy of binding, the Gibbs free energy $\left(\Delta \mathrm{G}^{\circ}\right)$ and entropy contributions ($\mathrm{T} \Delta \mathrm{S}$ ) to binding are calculated. Entropy driven interactions can indicate a strong effect of hydrophobic interactions and/or conformational changes, while enthalpy driven interactions can indicate a strong effect of hydrogen and van der Waals bonding.,11,12 Furthermore, with these experiments the stoichiometry of binding can be calculated to determine how many molecules of ligand bind to one enzyme molecule. In an ITC-MIM experiment, the reactant is titrated to the enzyme to establish a stepwise increase in reactant concentrations, mimicking the classical enzyme kinetics experiment. At each titration step, the energy produced by the enzyme reaction causes a stable shift of the signal, resulting in descending steps. This shift is directly proportional to the rate of the reaction, thereby enabling the calculation of $K_{m}$. However, the reaction enthalpy $\left(\Delta \mathrm{H}_{\text {reaction }}\right)$ and the turnover number $k_{\text {cat }}$ can only be determined using ITC-SIM experiments. Therein, reactant is titrated to a higher concentration of enzyme, than in the ITC-MIM experiment. This approach results in the signal returning to the baseline and the resulting peak is integrated to yield $\Delta \mathrm{H}_{\text {reaction. }}$ In a variation of this experiment, the "recurrent single injection experiment" (ITC-rSIM), ${ }^{9}$ insight on product inhibition or activation can be gained. In summary, ITC kinetics experiments can be used to determine the Michaelis Menten constant, $K_{m}$ and turnover number, $k_{c a t}$, based on the appropriate kinetic model. However, the acquisition of high-quality, reproducible data is a mandatory prerequisite to achieve robust modelling.

Any model is only as good as the data it is based on and it seems trivial that the most important criterion for "good data" is that it can be reproduced. However, although reproducibility of enzyme kinetic data is of such paramount importance for biocatalysis, it is inherently challenged by several factors, such as missing parameters of the experimental procedures, measurement artefacts and bad experimental setup. ${ }^{13-18}$ Hence overcoming the current "reproducibility crisis" ${ }^{19}$ will require the 
application of recommendations and guidelines for quality control. One important quality criterion for enzyme catalysis is the purity and "the homogeneity of an enzyme sample" (HES). ${ }^{18}$ While the purity of the sample is usually stable over the course of experiments, HES can change and lead to measurement artefacts which can be detrimental for highly sensitive methods such as ITC. HES can quickly be determined using dynamic light scattering (DLS) analysis. ${ }^{20}$ Furthermore, process parameters such as the reaction buffer and its precise electrolyte composition are known to affect enzyme activity. ${ }^{21}$ Especially in ITC experiments, the choice of a suitable buffer with low ionization enthalpy is crucial to improve the quality of the ITC experiment and the resulting data. ${ }^{7,22}$ Therefore, it has been established to ideally perform ITC experiments in different buffers in order to obtain bufferindependent data. ${ }^{23}$ Overall, the goal of reproducible experiments must be to provide a solid data foundation for robust modeling, ultimately enabling the effective application of data-based techniques, such as machine learning. Furthermore, as discussed above, the entire modeling process must be reproducible. To achieve this, standard operating procedures (SOPs), open and F.A.I.R. (findable, accessible, interoperable, and re-usable) data analysis and modeling workflows are critical.

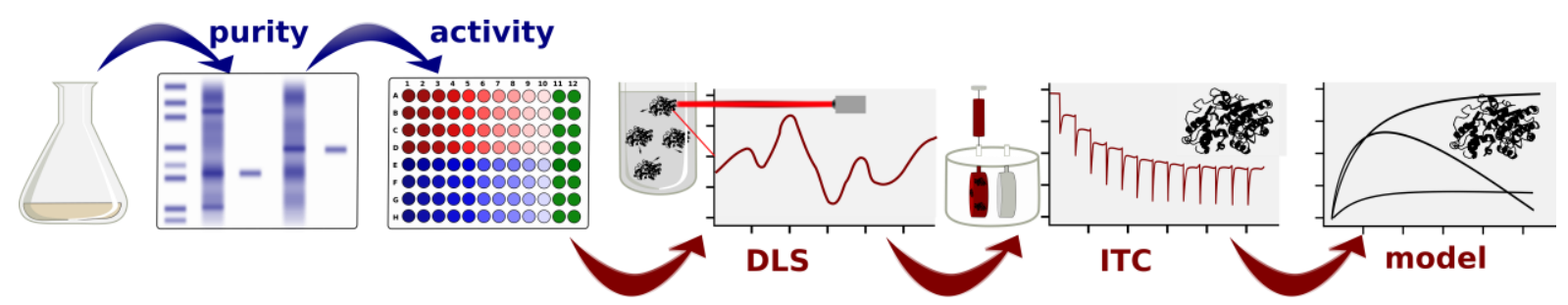

Figure 1: Workflow to create high-quality data for robust enzyme reaction modeling. Traditional approaches to enzyme kinetic data (blue) primarily use spectrophotometric activity measurements. The implementation of dynamic light scattering (DLS) and isothermal titration calorimetry (ITC) for quality control and mechanistic insights, respectively, leads to an increase in data quality to enable robust modeling.

To overcome the above limitations, we report here a methodological approach (Figure 1 ) to identify and implement relevant parameters that can improve the mechanistic understanding of enzymes and be used to acquire reproducible data for enzyme reaction modeling. To improve data quality and enable mechanism-based modeling in silico, DLS and ITC were implemented for quality control and mechanistic insights into the traditional enzyme kinetics workflow (Figure 1). To experimentally validate the performance of our proposed standardized workflow, we chose the NADPH-dependent ketoreductase Gre2p as a challenging, multi-reactant model system. Thus, we sought to address the challenge of investigating for the first time a complex multistep mechanism using a combined ITCbased binding and kinetics approach in conjunction with DLS. We found that our approach provides a complete picture of the binding and kinetic parameters relevant for enzyme catalysis. The usefulness of this approach was further demonstrated by the analysis of reactions in different buffer conditions, thus determining the previously incompletely understood mechanism of Gre2p. To enable reproducibly, we document our work by reporting standard operating procedures (SOPs), using programming workflows and storing all data in a FAIR repository (https://fairdomhub.org/investigations/464\#projects). 
Results and Discussion

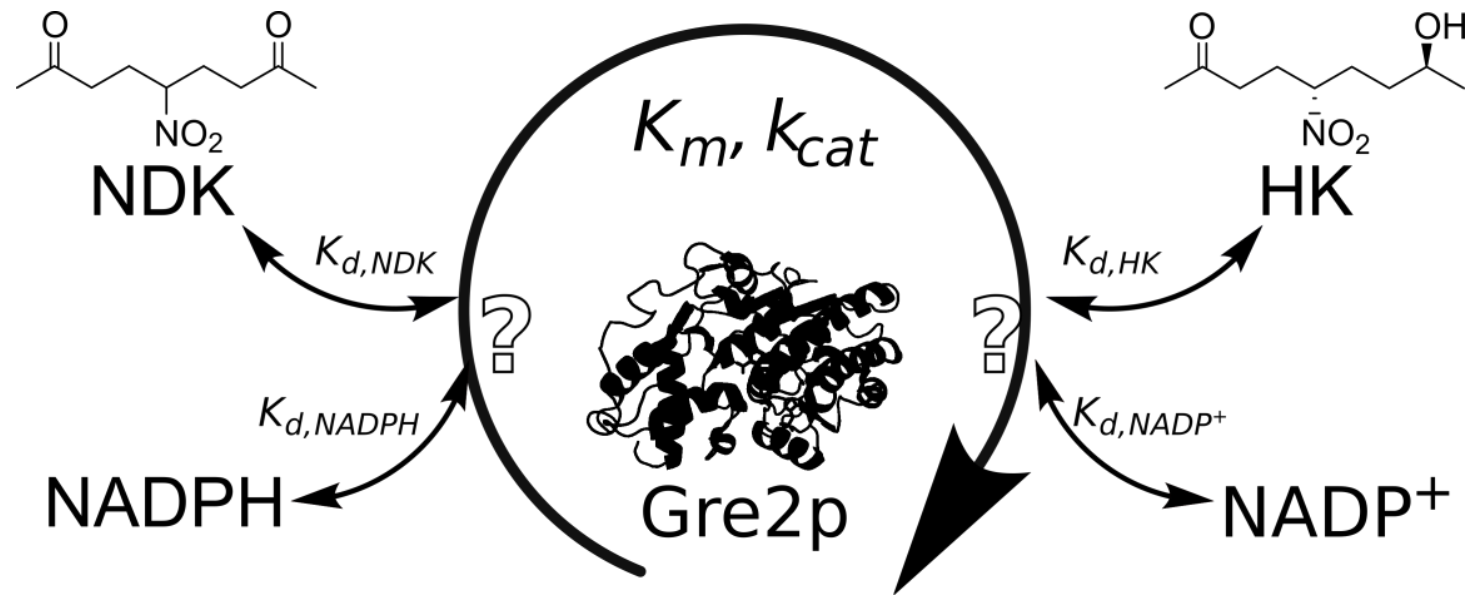

Figure 2: Gre2p catalyzed conversion of NDK. Gre2p requires the NADPH cofactor for catalysis and the conversion of 5nitrononane-2,8-dione (NDK) to the preferentially produced (5S,8S)-anti hydroxyketone (HK). Isothermal titration calorimetry (ITC) was applied for the determination of the binding and kinetic parameters shown: dissociation constants $\left(K_{d}\right)$ for the reactants (NADPH, NDK, NADP+, HK) and kinetic parameters (Michaelis Menten constant, $k_{m}$ and turnover number, $k_{c a t}$ ).

Introducing Gre2p

Many industrially relevant enzymes use cofactors, such as NADPH or FAD, ${ }^{24-26}$ and thus may form tri-molecular complexes. We chose the NADPH-dependent redox enzyme Gre2p as a challenging, multi-reactant model system to experimentally investigate the performance of our proposed workflow (Figure 1). The ketoreductase Gre2p (Genes de respuesta a estres (stress-response gene), EC 1.1.1.283) has been previously used in the asymmetric synthesis of chiral alcohols with excellent enantioselectivities. ${ }^{27,28}$ Based on the available crystal structures, 4PVD and 4PVC, and computational docking experiments, an induced fit mechanism has been proposed for NADPH binding. ${ }^{29}$ Beyond that, little is known about the detailed mechanism of this enzyme despite its application in relevant areas of biocatalysis. ${ }^{28,30-33}$ Kinetic parameters have been published for hexane-2,5-dione, hexane-(2S,5S)diol $^{27}$ and for a variant of Gre2p for nitrononane-2,8-dione (NDK, Table S1). ${ }^{32}$ In this work, we chose the NDK reaction (Figure 2 ) because it involves multiple reactants (NADPH, NDK, NADP ${ }^{+}$or (5S,8S)-anti hydroxyketone, HK) that may affect the reaction as they accumulate. The reaction can continue to the chiral diol, albeit much slower than the reaction to HK (Figure S1). Multi-reactant enzyme mechanisms are difficult to study with conventional techniques. Elucidating such mechanisms requires the determination of the binding order of the reactants, ${ }^{34-36}$ and the determination of inhibition modes by the reactants. Therefore, the use of ITC seemed worthwhile to gain mechanistic insights and to elicit the robustness of our approach.

We have used purified, heterologously expressed, hexahistidine(his)-tagged Gre2p from Saccharomyces cerevisiae (Sequence ID AJT71311.1, without the added his-tag) (Figure S2). The sequence of this Gre2p is identical to the Gre2p described by Müller et al. ${ }^{27}$, but differs from the Gre2p in the crystal structures 4PVD and 4PVC by six surface residues. Gre2p is dimeric in crystal structures due to crystal packing, but reported to be monomeric in solution. ${ }^{27,29,37}$ Assuming a monomeric Gre2p and the molecular weight of $39040 \mathrm{Da}$, this would correspond to a hydrodynamic radius of almost 3 $\mathrm{nm} .{ }^{38} \mathrm{DLS}$ measurements showed that our purified, his-tagged Gre2p has a hydrodynamic radius of approx. $6 \mathrm{~nm}$ (Figure S3). This larger than expected size is likely due to a certain amount of polydispersity in the sample and should not be interpreted as Gre2p being dimeric. In the following, 
we employed DLS to routinely monitor the quality of the sample by determining the HES in order to investigate whether experimental processes have an impact on enzyme agglomeration.

\section{Spectrophotometrically determined kinetic parameters}

To set a starting point for our study, the activity of recombinant Gre2p was determined spectrophotometrically by measuring the change in NADPH absorbance at $340 \mathrm{~nm}$ during the conversion of hexane-2,5-dione and NDK (Table 1). For hexane-2,5-dione (Table 1 and Figure S4a), we obtained values that were in good agreement with previously published data ${ }^{27}$ (Table S1). However, we found that our data were best represented by a substrate inhibition model (eq. 2, Figure S4b), which resulted in higher $K_{m}$ and a lower $k_{\text {cat }}$ compared to values obtained with the classical MichaelisMenten model (Table 1). For NDK, no substrate inhibition could be observed (Figure S4c, Table 1). It is important to note that substrate inhibition is only identifiable from the raw data (Figure S4). Therefore, important information may be lost if only kinetic parameters are reported. Also, these classical spectrophotometric assays can quickly be NADPH-limited due to the upper detection limit of NADPH at 0.3-0.5 mM. If $K_{d, N A D P H}$ is in the same range as $K_{m}$ (Table 1), these NADPH concentrations are too low to saturate Gre2p with NADPH, and the kinetic parameters thus measured can only serve as rough estimates. Knowledge of the $K_{d}{ }^{\prime} s$ of the reactants would help to solve this issue and would shed further light on the reaction mechanism of Gre2p. Also, the apparent absence of substrate inhibition in the NDK reaction calls for further analyses. Thus, the previous experiments clearly show gaps in knowledge that can be filled with ITC.

Table 1: Comparison of kinetic parameters determined spectrophotometrically (UV-Vis) and with ITC-MIM, fitted using the classical Michaelis Menten model (eq. 1) or a substrate inhibition model (eq. 2). See Figures $\mathbf{S} \mathbf{4}$ and $\mathbf{5}$ for the plotted raw data. Details on the ITC measurements are given in the section "Determination of the kinetic parameters with ITC". $V_{\max }$ was converted to turnover numbers ( $\mathrm{k}_{\text {cat }}$ ), assuming a molecular weight for Gre2p of 39040 Da (eq. S1).

\begin{tabular}{|l|l|l|l|l|l|}
\hline Method & Substrate & Model & $K_{m}(\mathrm{mM})$ & $k_{\text {cat }}\left(\mathrm{s}^{-1}\right)$ & $K_{i}(\mathrm{mM})$ \\
\hline UV-Vis & hexane-2,5-dione & Michaelis Menten, eq. 1 & $1.4 \pm 0.6$ & $4.1 \pm 0.5$ & Not applicable \\
\hline UV-Vis & hexane-2,5-dione & Substrate inhibition, eq. 2 & $2.5 \pm 1.0$ & $5.4 \pm 0.7$ & $99 \pm 8$ \\
\hline UV-Vis & NDK & Michaelis Menten, eq. 1 & $2.4 \pm 0.6$ & $1.8 \pm 0.1$ & Not applicable \\
\hline ITC-MIM & NDK & Michaelis Menten, eq. 1 & $0.7 \pm 0.2$ & $2.7 \pm 0.8$ & Not applicable \\
\hline ITC-MIM & NDK & Substrate inhibition, eq. 2 & $1.4 \pm 0.1$ & $3.6 \pm 0.9$ & $10^{*} \mathrm{~K}_{\mathrm{m}}{ }^{\#}$ \\
\hline
\end{tabular}

\#fixed during fitting based on the estimation of $\mathrm{K}_{\mathrm{d}, \mathrm{NDK}}$ from the ITC-BIND experiments

\section{Binding of reactants to Gre2p}

Since the results from the above spectrophotometric experiments called for additional investigations on the binding parameters of the reactants, ITC-BIND experiments were performed, where one reactant was titrated to Gre2p to determine $K_{d}, \Delta H_{\text {binding, }} \Delta G^{\circ},-T \Delta S$ and the stoichiometry of binding. These experiments can also shed light on the reaction mechanism by determining the binding order of the reactants. In an ordered, sequential mechanism, only one reactant will bind to the free enzyme, while in a non-sequential mechanism, both reactants will bind to the free enzyme, thus allowing the distinction of these mechanisms. 

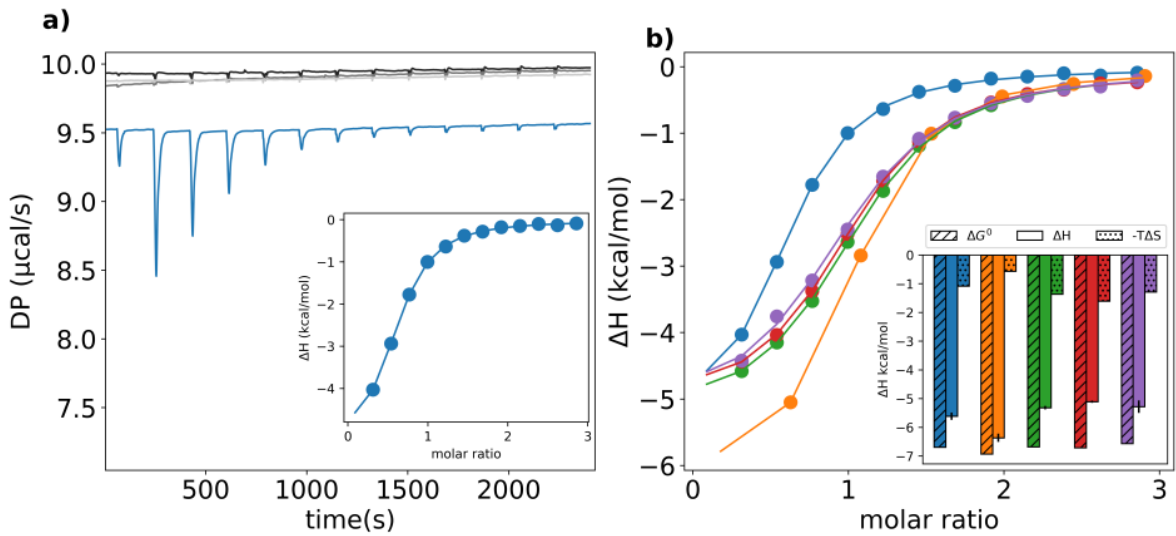

Figure 3: ITC-BIND experiment to determine the binding parameters of NADPH to Gre2p. Binding of NADPH is much stronger than expected from the Michaelis constant obtained from spectrophotometric measurements $\left(K_{m}=2.4 \mathrm{mM} v s K_{d}=12.2 \mu M\right)$. a) representative data of the raw heat of the titrations of NADPH to Gre2p (blue line), from which isotherms are integrated (inset, dots) and $K_{d}$ is fitted (inset, line). The heat of dilution of the controls (NADPH to buffer, buffer to Gre2p and buffer to buffer as represented by black, grey and light-grey titrations, respectively) is negligible compared to the heat of binding. $b$ ) integrated isotherms (dots) and fits for $K_{d}$ (lines) of five replicates in blue, orange, green, red and purple as a function of the molar ratio of NADPH and Gre2p; the inset shows the resulting energy terms, the experimental Gibbs free energy $\left(\Delta G^{\circ}\right.$, hatched), enthalpy ( $\triangle H$, blank) and entropy (-TSS, dotted). Errors are only given on the directly measured enthalpy values. Note that the slope at the inflection point corresponds to $1 / K_{d}$ indicating that the molar ratio at the inflection point corresponds to the stoichiometric ratio.

Table 2: Binding parameters obtained with ITC-BIND experiments for Gre2p with NADPH or NADP+ in KP, buffer, $p H 7.5$, at $25^{\circ} \mathrm{C}$.

\begin{tabular}{|l|l|l|l|l|}
\hline Ligand & $\begin{array}{l}K_{d} \\
(\mu \mathrm{M})\end{array}$ & $\begin{array}{l}\Delta \mathrm{G}^{0} \\
(\mathrm{kcal} / \mathrm{mol})\end{array}$ & $\begin{array}{l}\Delta \mathrm{H} \\
(\mathrm{kcal} / \mathrm{mol})\end{array}$ & $\begin{array}{l}-\mathrm{T} \Delta \mathrm{S} \\
(\mathrm{kcal} / \mathrm{mol})\end{array}$ \\
\hline $\mathrm{NADPH}$ & $12.2 \pm 2.5$ & $-6.7 \pm 0.1$ & $-5.5 \pm 0.5$ & $-1.2 \pm 0.4$ \\
\hline $\mathrm{NADP}^{+}$ & $96.6 \pm 18.0$ & $-5.5 \pm 0.1$ & $-4.3 \pm 1.0$ & $-1.2 \pm 1.2$ \\
\hline
\end{tabular}

ITC-BIND experiments with NADPH (Figure 3) measured a $K_{d, N A D P H}$ in the lower micromolar range (Table 2). Although this is an almost 1000-fold difference compared to the spectrophotometrically determined $K_{m}$ in the low millimolar range (Table 1 ), the result seemed reasonable because it is known that NADPH often binds much more strongly to enzymes with the same EC number as Gre2p (EC 1.1.1.283). ${ }^{39-42}$ ITC-BIND experiments further elucidated that binding of NADPH to Gre2p is dominated by enthalpy, indicating that favorable hydrogen and/or van der Waals bonding drive the binding process (Table 2). The fact that entropy plays an unfavorable role in NADPH binding suggests that an energetically unfavorable conformational change has to occur to allow NADPH binding. Such an interpretation is supported by previously reported crystal structures that reveal a conformational change induced by NADPH binding. ${ }^{29}$ Finally, stoichiometry of binding is another binding parameter routinely obtained with ITC-BIND experiments. Stoichiometry of binding for NADPH, as determined from the inflection point of the binding curve, indicated that one molecule of NADPH binds to one active site of Gre2p $(0.9 \pm 0.2$, Figure 3$)$. This was to be expected from the fact that Gre2p is monomeric in solution and contains one active site per monomer. ${ }^{27,29,37}$ In contrast, binding of NDK to Gre2p was not detectable by ITC (Figure S5). Due to solubility limitations of NDK in the buffer, a maximum concentration of $100 \mathrm{mM}$ NDK could be used for titrations. We therefore estimate that binding of NDK to Gre2p is at least 10-times weaker than the spectrophotometrically determined $K_{m}$ would imply (Table 1 ). Therefore, binding of NDK to Gre2p without NADPH bound ( $K_{d,}$ 
NDK, Gre2 $\left.2 p_{\text {apo }}\right)$ is at least 10-times less favorable than binding of NDK to Gre2p with NADPH bound ( $K_{m}$, Gre2 $\left.p_{\text {holo }}\right)$. These data consistently show that Gre2p operates via an ordered, sequential mechanism in which NADPH binds first, resulting in Gre2 $p_{\text {holo, }}$ and that only Gre2 $p_{\text {holo }}$ can then bind NDK (Figure 4). Indeed, previously reported crystal structures that show a conformational change due to the induced fit NADPH binding ${ }^{29}$ are also supportive of this mechanism since they indicate that indeed two distinct structural conformations of Gre2p exist (Gre2p $p_{\text {apo }}$ and Gre2p $p_{\text {holo }}$ ).

The binding parameters of NADP ${ }^{+}$were also determined by ITC-BIND experiments (Table 2, Figure S6). Binding of $\mathrm{NADP}^{+}$was almost 10-times weaker than binding of NADPH (Table 2). Compared to $N A D P H$, the Gibbs free energy $\left(\triangle G_{\mathrm{NADP}+}^{\circ}\right)$ was less favorable (less negative), with less favorable enthalpy contribution $\left(\Delta \mathrm{H}_{\mathrm{NADP}+}\right)$, and comparable entropy contribution (-T $\triangle \mathrm{S}_{\mathrm{NADP}+}$, Table 2 ). This suggests that the binding of $\mathrm{NADP}^{+}$to Gre2p is driven by favorable hydrogen and van der Waals bonding, similar as the binding of NADPH. It has to be noted, however, that due to the weak binding, the fit of NADP+ binding is of low quality as the data result in a c-value close to 1.0 (Table S2). The cvalue (eq. $\mathbf{S 2}{ }^{43}$ ) depends on the binding affinity, the number of binding sites and the heat of binding $(\Delta \mathrm{H})$ and it should be in the range of 1-1000 to ensure appropriate sample concentration in the ITCBIND experiment. ${ }^{44}$ Although the low c-value and the corresponding $K_{d, \text { NADP }}$ could have a considerable error, no further experimental clarification could be performed, since unattainably high enzyme concentrations would have to be used to obtain data with a potentially higher c-value. Binding of HK to Gre2p was very weak and could only be estimated to be at least as weak as binding of NDK (Figure S7). The order of unbinding of $\mathrm{HK}$ and $\mathrm{NADP}^{+}$cannot be determined experimentally and would necessitate computational approaches, such as molecular modelling.

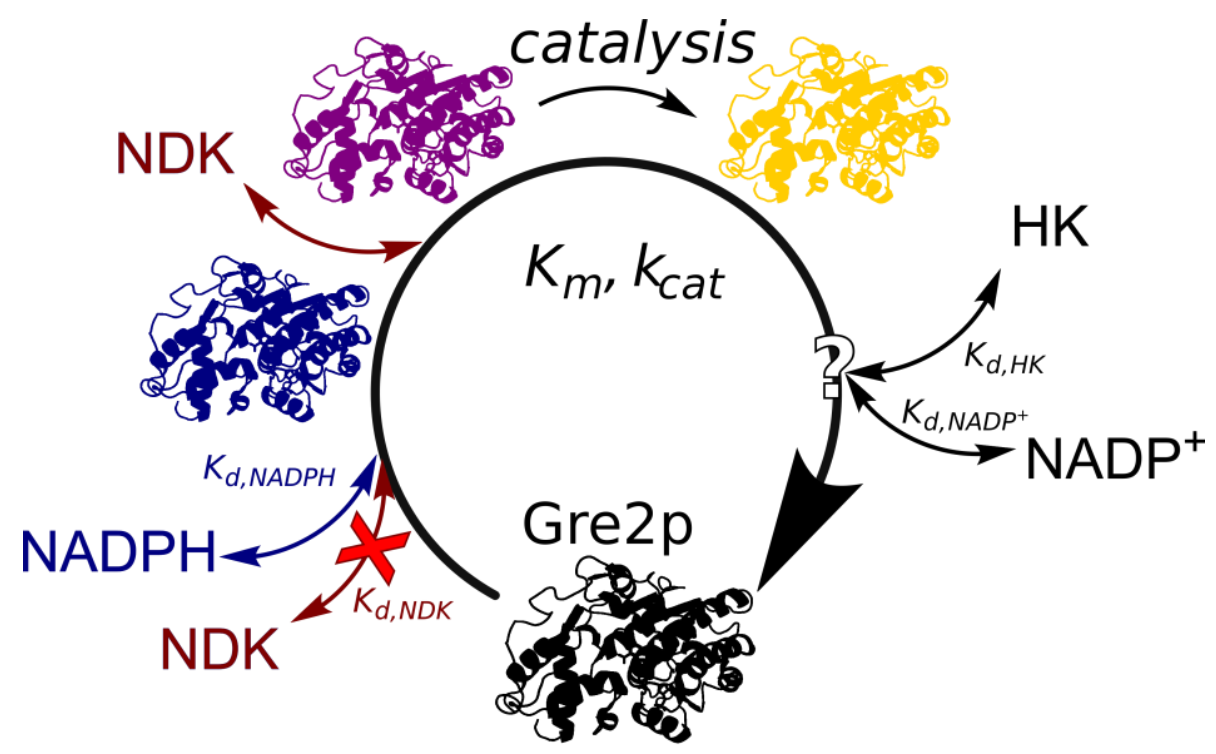

Figure 4: The proposed mechanistic model for Gre2p. The black structure represents Gre2p apo, blue Gre2p holo, purple fully reactive Gre2p with both NADPH and NDK bound, yellow Gre2p after the reaction with both NADP+ and HK bound. The crossed arrow (red) indicates that NDK cannot bind to Gre2p unless NADPH is already bound (Gre2p holo). Binding of NDK to Gre $2 p_{\text {holo }}$ is represented in part by $K_{m}$. Note that the order of unbinding of $H K$ and $N A D P^{+}$cannot be determined experimentally.

\section{Determination of the kinetic parameters using ITC}

With our experimentally obtained details on the binding parameters of all reactants, we then investigated the kinetics of Gre2p with ITC-MIM and ITC-rSIM experiments to determine accurate 
kinetic parameters and further elucidate the reaction mechanism. The ITC-MIM experiments were initially fitted using a standard Michaelis-Menten model to determine the kinetic parameters $\left(K_{m}\right.$ and $k_{\text {cat }}$ ) (Table 1, Figure 5a). We found that $K_{m}$ was more than 3-fold lower than the value obtained from spectrophotometric measurements, whereas $k_{\text {cat }}$ increased slightly (Table 1$)$. This indicated that, despite $K_{d, \text { NADPH }}$ being $<<0.3 \mathrm{mM}$, Gre2p is not saturated with NADPH in the spectrophotometric assay. However, the reaction rate decreased unexpectedly in the ITC-MIM experiments (Figure $\mathbf{5 b}$ ). In such an ITC-MIM experiment, the substrate concentration inside the cell increases with each subsequent titration step and, thus, high substrate concentrations are reached in the late phases of the experiment. Three different hypotheses could explain the observed decrease in reaction rate. Firstly, measurement artifacts in the ITC-MIM experiment, secondly enzyme inactivation towards the end of the ITC experiment, and, thirdly, a slow-onset inhibition by product or substrate inhibition may occur. Product inhibition would be caused by the product accumulating due to the enzymatic reaction, while substrate inhibition would be the result of the substrate accumulating at higher concentrations with the increasing number of ITC titration steps.

a)

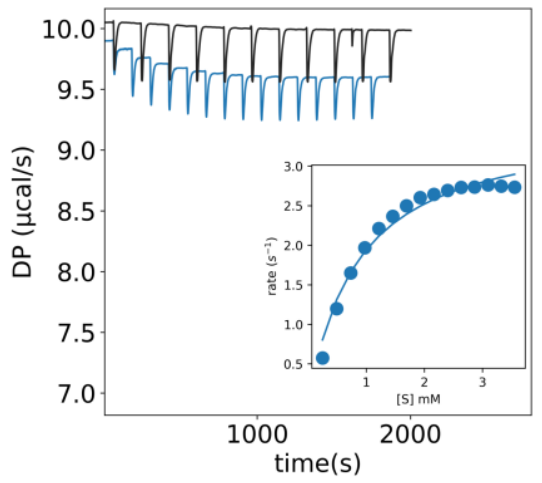

b)

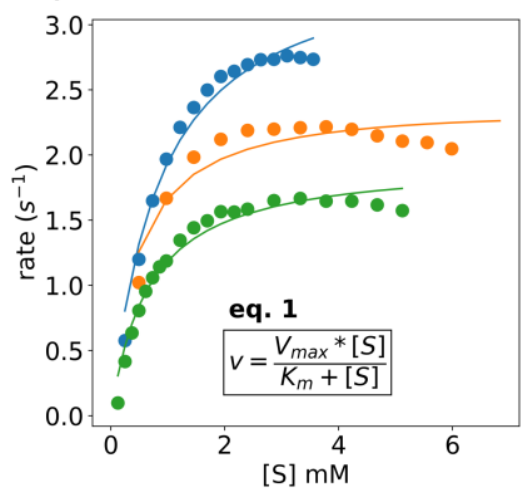

c)

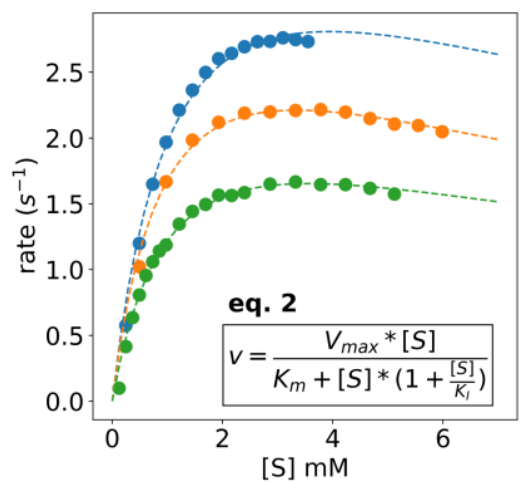

Figure 5: Kinetics for Gre2p from ITC-MIM experiments indicate that the classical Michaelis-Menten model (eq 1.) does not represent the data well and that data are best fitted using a substrate inhibition model (eq. 2). Three replicates in blue, orange and green are shown, fitted with different kinetic models in b) and c). a) representative data of the raw heat of the titrations of NDK to a solution of NADPH and Gre2p in the cell (blue line), from which isotherms are integrated (inset, dots) and $K_{m}$ is fitted using the standard Michaelis-Menten model (inset, lines, eq. 1). The control reaction (titrating buffer to Gre2p and $N A D P H$ in the cell, black) shows that the baseline is stable compared to the heat produced in the reaction, leading to descending steps (blue). Note that the time on the $x$-axis in a) is proportional to the substrate concentration on the $x$-axis in the inset and in b) and c) because subsequent injections every $180 \mathrm{~s}$ lead to a stepwise increase in substrate concentration. $b$ ) integrated isotherms (dots) and fits for $K_{m}$ and $k_{\text {cat }}$ (lines). Note that the standard Michaelis-Menten model does not represent the data well. c) integrated isotherms (dots) and fits for $K_{m}$ and $k_{\text {cat }}$ (lines) using a substrate inhibition model (eq. 2, details in main text). For $b$ ) and c), the maximal reaction rate, $k_{c a t}$, was calculated using $\Delta H_{\text {reaction }}$ obtained from ITC-rSIM experiments (Table S4, Figure S19), and varies between replicates (Table 1). Kinetic parameters resulting from these models are summarized in Table 1. Note that the difference in kinetic parameters measured by ITC and spectrophotometry indicates that NADPH is indeed limiting in spectrophotometric assays (Table 1).

We initially looked into possible measurement artifacts in ITC-MIM experiments. Specifically, we tested the order in which reactants are titrated to Gre2p, the enzyme concentration and the stirring speed. ${ }^{9,45}$ While we could clarify that the order of reagent injection can lead to artifacts (Figure S8), the procedure specified in the SOP (NDK is titrated to a mixture of NADPH and Gre2p) does not show such artifacts (Figure 5a). Another artifact, that is known to be caused by a too high the enzyme concentration, can lead to "steps sloping towards the baseline". ${ }^{9}$ Although we could specifically induce this artifact, it is a systematic error that cannot explain the observed phenomenon of the decrease in reaction rate at the end of the reaction (Figure S9). Finally, mechanical stress due to stirring in the ITC- 
MIM experiment could lead to enzyme agglomeration and inactivation, which would become more significant in late stages of the experiment. ITC experiments carried out with identical samples in two individual instruments at different stirring speeds indicated that the slower stirring speed led to noisier and lower quality data (Figures S10, S11) as compared to the faster stirring speed (Figures S8, S9 and S12). More details on all three possible artifacts and our tests can be found in the SI section "Elimination of measurement artefacts in the ITC-MIM experiments". Altogether, these tests excluded measurement artifacts, leaving the hypotheses of enzyme inactivation and slow-onset inhibition to be tested further.

Enzyme inactivation in the ITC was tested by performing DLS and activity measurements before and after mock ITC experiments and stirring or shaking in Eppendorf tubes (Figure S13). In these mock experiments, only buffer was titrated to Gre2p. Likewise, no reactants were present in any of the other experiments to determine HES of Gre2p described below. As discussed above (Figure S3), DLS data showed that Gre2p was already heterogeneous prior to any treatment and even directly after the first freeze/thaw cycle, as indicated by the high Z-averages and polydispersity indices (Figure S13). Samples after treatments showed that the Z-average increased by up to two orders of magnitude compared to the sample before treatment (Figure 13a). Accordingly, the PDI value also increased after treatments, although less pronounced (Figure 13b). HES was severely decreased by the mock ITC experiments and even by stirring in Eppendorf tubes, but much less severely during mere shaking in Eppendorf tubes. Hence, intensive stirring caused the deterioration of HES in the ITC, presumably by induction of agglomeration of the enzyme due to mechanical sheer forces.

As a possible means to reduce the agglomeration of proteins, we tested ITC measurements in the presence of the nonionic detergent Tween-20 (Tween), a widely used additive to prevent nonspecific protein binding and aggregation. Indeed, the presence of Tween resulted in a more homogeneous enzyme sample as compared to the sample in the absence of Tween (Figures S13a, b). Unfortunately, however, Tween also led to the loss of more than $50 \%$ of the activity (Figure S13c). Additional investigation of this phenomenon by ITC indicated that Tween had a detrimental effect on the binding of NADPH to Gre2p, leading to a 3 -fold increase in $K_{d}$ (Table S3 and Figure S14). Furthermore, we established by ITC-MIM experiments that $K_{m}$ was increased and $k_{\text {cat }}$ decreased in the presence of $0.1 \%$ Tween (Table S3 and Figure S15) as compared to the parameters obtained in the absence of Tween. Given the adverse effect of Tween on Gre2p's kinetic and thermodynamic parameters, we did not proceed with Tween in the buffer.

We then tested whether the observed decrease in HES is linked to changes in Gre2p activity. Using spectrophotometry, we analyzed the activity of Gre2p after different treatment steps, i.e., the initial freeze/thaw cycle, the mock ITC experiment, a $1 \mathrm{~h}$ incubation at room temperature or a $5 \mathrm{~h}$ incubation on ice (Figure S16). After these treatments, reactants were added and the activity was immediately determined by monitoring the NADPH absorbance. We found a $25 \%$ loss of activity after the mock ITC experiments (Figure S16). A comparable activity loss (29\%) was also measured after storing Gre2p at room temperature (Figure S16). While these comparable losses in activity suggested that Gre2p inactivation was prone to thermal instability, in-depth analysis by employing a Selwyn test ${ }^{46}$ provided evidence that slow-onset inhibition rather than thermal inactivation is the reason for the observed reduction in activity at later reaction stages. This hypothesis was supported by ITC-rSIM experiments, which indicated that very weak to no enzyme inactivation occurred in the ITC (Figure 
S19). More details can be found in the SI sections "Specific activity and Selwyn test" and "Recurrent single injection experiments".

While the distinction between enzyme inactivation and slow-onset inhibition is notoriously difficult with a Selwyn test, it can be achieved with ITC-rSIM experiments. If the injection depth of the peaks and $\Delta \mathrm{H}_{\text {reaction }}$ of all recurrent injections are identical, no product inhibition is present, whereas enzyme inactivation leads to broader peaks in ITC-rSIM experiments. Analysis of the ITC-rSIM data showed that peaks of both injections had identical depth (Figure S19) and $\Delta H_{\text {reaction }}$ did not decrease in the second injection (Table S4), thus indicating that product inhibition was absent. Furthermore, fitting ITC-MIM data with a model assuming competitive product inhibition (eq. S3) and using $K_{d, \text { NADP+ }}$ (from Table 2) as $K_{i}$ did not improve the quality of the fit, thus contradicting the possibility of product inhibition.

After we could exclude all these possibilities, an investigation of the substrate inhibition remained, which had previously been observed in photometric assays for Gre2p conversion of hexane2,5-dione (Figure S4b) but not for NDK (Figure S4c). The same kinetic model for substrate inhibition (eq. 2) was used, and this model led to a very good fit of the ITC-kinetic data (Figure 5c) with the estimated $K_{i}=K_{d, N D K}=10^{*} K_{m}$ (Table1). Therefore, our experiments so far showed that Gre2p uses an ordered, sequential mechanism for the reaction with NDK (Figure 4), and that substrate inhibition must be included for reactions with NDK and hexane-2,5-dione to model kinetic data of Gre2p (eq. 2 and Figure 5). Of note, these insights would not have been possible using only spectrophotometric assays but indeed required the combination of ITC binding and kinetic experiments with supporting characterization of the HES by DLS.

Impact of process parameters on the enzyme mechanism

All experiments so far were performed in one reaction buffer ( $\mathrm{KP}_{\mathrm{i}}$ buffer, $100 \mathrm{mM}, \mathrm{pH}$ 7.5). However, the reaction buffer is a generally important process parameter for biocatalytic applications, since the buffer's electrolyte composition can markedly affect enzyme activity, ${ }^{21,47}$ a fact that is also revealed by the buffer influence on the results of ITC experiments. ${ }^{7,22}$ Therefore, we investigated whether we could characterize the influence of the reaction buffer on enzyme kinetics with our work flow. To this end, the whole series of the above discussed DLS and ITC experiments were conducted in two additional buffers, PBS (1x PBS, pH 7.5) and HEPES (100 mM, pH 7.5). DLS experiments indicated that the homogeneity of Gre2p was comparable in all buffers tested (Figure S20). The results of the 
ITC experiments, carried out following the previously set SOPs, are summarized in Figure 6 and Tables S5, S6.
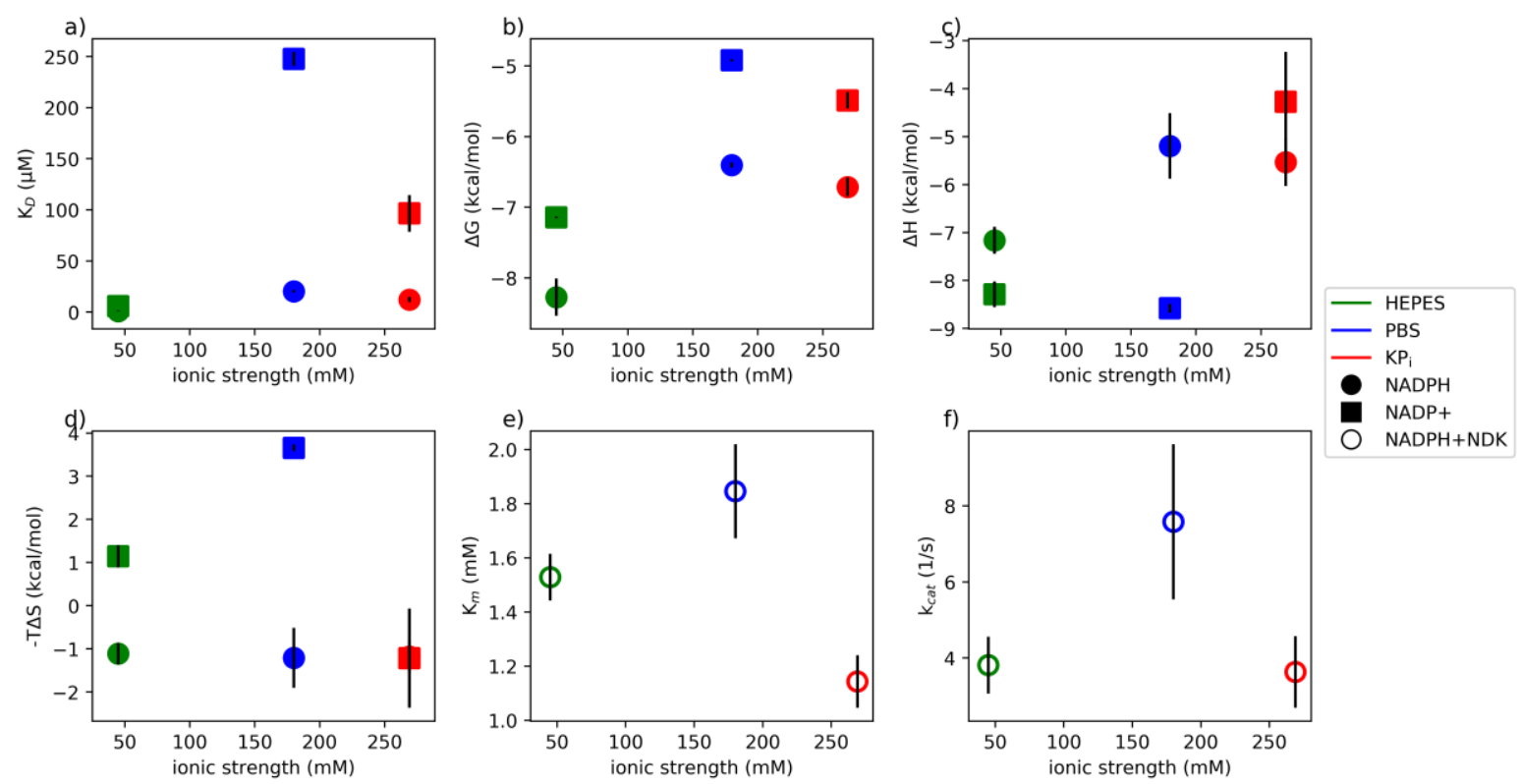

Figure 6: The reaction buffer impacts kinetic and binding parameters. Overview of these parameters as a function of the ionic strength of the buffer ( $K P_{i}, P B S$ or HEPES, red blue and green). Binding parameters for NADPH and NADP+ are shown as circle and square, respectively. a) dissociation constants $K_{d}$ b) Gibbs free energy of binding, $\Delta G^{\circ}$ c) Enthalpy contribution to binding, $\triangle H$ d) Entropy contribution to binding, $-T \Delta S$ e) Michaelis constant $K_{m} f$ ) turnover number $k_{\text {cat }}$. See $S I$ for raw data for binding of NADPH and NADP' in PBS buffer (Figures S21, S22) and in HEPES buffer (Figures S23, S24), respectively. SI also contain the raw kinetic data for the reaction in PBS buffer (Figure S25) and in HEPES buffer (Figure S26). Note that $K_{d}$ values for NADPH and $N A D P^{+}$binding and associated entropy values are almost identical in some cases, thus leading to overlapping data points in a) for HEPES buffer (green) and d) for KP $i$ buffer (red). See Table $\mathbf{5}$ for all values.

Comparison of the binding parameters for NADPH and NADP ${ }^{+}$in $\mathrm{KP}_{\mathrm{i}}, \mathrm{PBS}$ and HEPES buffer obtained by ITC-BIND experiments indicated that $K_{d}$ for NADP ${ }^{+}$was 10 -times lower than for NADPH in $\mathrm{KP}_{\mathrm{i}}$ and PBS buffer (Figure 6a). While the difference in Gibbs free energy of binding NADPH and NADP ${ }^{+}$ was comparable in all buffers, (Figure $6 \mathbf{b}$ ), contributions of enthalpy (Figure $\mathbf{6 c}$ ) and entropy (Figure 6d) varied substantially. In HEPES and PBS buffer, the enthalpic term dominated NADPH binding (Figure 6c), whereas the entropic term dominated $\mathrm{NADP}^{+}$binding (Figure 6d). The difference in enthalpy and entropy contribution between NADPH and NADP+ binding was most pronounced in PBS buffer (Figures $6 c$, d). It should be noted, however, that due to the weak binding, the fit of NADP ${ }^{+}$binding in PBS buffer was of low quality, resulting in a c-value below 1.0 (eq. S2, Table S2). Nevertheless, these striking differences in binding parameters in these different buffers raised the question of whether and how kinetic parameters would be affected.

To test whether the observed buffer-dependent differences in binding behavior led to a change in kinetic parameters, ITC-MIM and ITC-rSIM experiments were performed and the resulting data was again fitted with the substrate inhibition model (eq.2, Figures S25, S26). The obtained $K_{m}$ and $k_{\text {cat }}$ values were highest in PBS buffer (Figures 6 e, $\mathbf{f}$, respectively and Table S6). ITC-rSIM experiments showed that product inhibition occurs in PBS buffer but not in $\mathrm{KP}_{\mathrm{i}}$ and HEPES buffer (Figure $\mathbf{S 1 9}$ and Table S4). Therefore, these experiments provided conclusive evidence that the buffer can change the mode of inhibition of Gre2p from substrate to product inhibition. Furthermore, the catalytic efficiencies $\left(\frac{k_{c a t}}{K_{m}}\right.$, Table S6) indicated that product inhibition in PBS buffer was less detrimental than 
substrate inhibition in the other two buffers. In fact, it was found that catalytic efficiency was about 1.7-fold greater in PBS $\left(4.2 \mathrm{mM} / \mathrm{s}\right.$ ) than in $\mathrm{KP}_{i}$ and HEPES (about $2.5 \mathrm{mM} / \mathrm{s}$ ) buffer. These results nicely illustrate that the detailed knowledge of thermodynamic and kinetic parameters as well as of the enzyme mechanism might be exploited to readily identify optimal buffers for a biocatalytic reaction by rational process engineering.

\section{Conclusion}

The identification of critical parameters for rapid optimization of biocatalytic processes is of paramount importance for rational process engineering. We have here introduced a standardized workflow that can be used for in-depth characterization of thermodynamic and kinetic parameters to yield insights into mechanistic details of enzyme catalysis under variable reaction conditions. Our workflow uses DLS for sample quality control as well as ITC and spectrophotometric activity measurements to determine thermodynamic and kinetic parameters. As illustrated for the cofactordependent Gre2p, the workflow is capable of uncovering enzyme inactivation and slow-onset inhibition and translating them into traceable, reproducible, high-quality data and models of the reaction mechanism. To enable the full reproduction of our results, we have followed established guidelines and best practices for SOPs ${ }^{48}$ and the reporting of enzyme and biocatalytic data ${ }^{18,49}$ in addition to storing our data on FAIRDOMHub (https://fairdomhub.org/investigations/464\#projects). ${ }^{50}$ Hence the work is in compliance with various large-scale efforts to tackle the current reproducibility crisis $^{19}$ such as NFDI4chem, NFDI4cat, NFDI4ing and the GO FAIR initiative. ${ }^{51-54}$

To illustrate the usefulness of our workflow under variable reaction conditions, we studied a cofactor-dependent enzyme that presented a new challenge for ITC methodology. Our approach revealed that the NADPH-dependent alcohol dehydrogenase Gre2p uses an ordered, sequential mechanism, the enzyme suffers from "slow-onset inhibition" and the mode of inhibition changes with the composition of the reaction buffer. The latter findings are of particular relevance for process engineering as they emphasize that a comprehensive understanding of the enzyme mechanism can be exploited to improve biocatalytic production processes. Our results suggest that solvent engineering approaches ${ }^{55,56}$ to optimize enzyme activity in biocatalytic processes should not be limited to organic solvents but also include aqueous buffer solutions. Therefore, the work should motivate further studies, for example, on the influence of the ionic strength of the buffer and its electrolyte composition, such as Hofmeister effects, ${ }^{57,58}$ on the mechanism of enzymes and thus on the overall biocatalytic process. The work also calls for further ITC-studies on enzyme variants for fundamental studies to identify the involvement of positions of interest on reactant binding or conversion. ${ }^{59}$ Finally, molecular modelling of the binding of reactants can provide atomistic insight into binding processes. ${ }^{60-}$ 63 Because our approach is amenable to automation and scale-up for high-throughput, the combination of such diverse approaches will provide the high-quality data needed for the engineering of enzymes and biocatalytic processes through machine learning to speed up the future development of industrial biocatalysis. ${ }^{1,64-67}$ 


\section{Experimental Section}

Detailed materials and methods, SOPs, raw data and files can be found in the SI and on FAIRDOMHub (https://fairdomhub.org/investigations/464\#projects).

\section{Acknowledgements}

The authors thank T. Peschke and M. Peng from IBG1 for kindly providing the PET22 GRE2-His vector, B. Huber from the Soft Matter Synthesis Laboratory at KIT for measuring NDK and HK mass spectra, and B. Tartsch, A. Rosenthal, N. Markova and P. Hofmann (all from Malvern Panalytical GmbH) for excellent technical support with DLS and ITC.

Funding sources: Funded by the Federal Ministry of Education and Research (BMBF) and the BadenWürttemberg Ministry of Science as part of the Excellence Strategy of the German Federal and State Governments. The work was also supported through the Helmholtz program "Materials Systems Engineering" under the topic "Adaptive and Bioinstructive Materials Systems".

The authors declare no competing financial interest.

\section{Supporting Information}

Data can be traced consistently from the plot in our publication to the raw data to the SOP used to generate the data with FAIRDOMHub (https://fairdomhub.org/investigations/464\#projects).

Ott_GYGLI_2021_SI.pdf contains supplementary figures, materials and methods section as well as other details on information mentioned in the main text

SOPs.zip contains experimental SOPs (as pdfs) referenced in Ott_GYGLI_2021_SI.pdf with detailed information on how experiments were performed

python_ITC.zip contains the ITC data and the python workflow developed in this work to analyze ITC data and model with custom kinetic models (.py, .csv, .apj and .png)

python_DLSandSpAc.zip contains the raw data from the specific activity and stability experiments (UVVis and DLS), details in SI.pdf in section "Enzyme Inactivation" and the script used to create Figures S3, S13, S16, and S20.

python_Selwyntest.zip contains the raw data from the specific activity experiments (UVVis) details in SI.pdf in section "Specific activity and Selwyn test" and the script used to create Figure S17.

pET22 GRE2-His.txt contains the full vector sequence used for expression of Gre2p in fasta format 


\section{References}

(1) Woodley, J. M. Accelerating the Implementation of Biocatalysis in Industry. Appl. Microbiol. Biotechnol. 2019, 103, 4733-4739. https://doi.org/10.1007/s00253-019-09796-x.

(2) Woodley, J. M. New Frontiers in Biocatalysis for Sustainable Synthesis. Curr. Opin. Green Sustain. Chem. 2020, 21, 22-26. https://doi.org/https://doi.org/10.1016/j.cogsc.2019.08.006.

(3) Adams, J. P.; Brown, M. J. B.; Diaz-Rodriguez, A.; Lloyd, R. C.; Roiban, G.-D. Biocatalysis: A Pharma Perspective. Adv. Synth. Catal. 2019, 361, 2421-2432.

https://doi.org/https://doi.org/10.1002/adsc.201900424.

(4) Planas-Iglesias, J.; Marques, S. M.; Pinto, G. P.; Musil, M.; Stourac, J.; Damborsky, J.; Bednar, D. Computational Design of Enzymes for Biotechnological Applications. Biotechnol. Adv. 2021, 47, 107696. https://doi.org/10.1016/j.biotechadv.2021.107696.

(5) Roda, S.; Santiago, G.; Guallar, V. Mapping Enzyme-Substrate Interactions: Its Potential to Study the Mechanism of Enzymes. Adv. Protein Chem. Struct. Biol. 2020, 122, 1-31. https://doi.org/10.1016/bs.apcsb.2020.06.001.

(6) Wu, S.; Snajdrova, R.; Moore, J. C.; Baldenius, K.; Bornscheuer, U. T. Biocatalysis: Enzymatic Synthesis for Industrial Applications. Angew. Chemie Int. Ed. 2021, 60, 88-119. https://doi.org/https://doi.org/10.1002/anie.202006648.

(7) Falconer, R. J. Applications of Isothermal Titration Calorimetry - the Research and Technical Developments from 2011 to 2015. J. Mol. Recognit. 2016, 29, 504-515. https://doi.org/https://doi.org/10.1002/jmr.2550.

(8) Freyer, M. W.; Lewis, E. A. Isothermal Titration Calorimetry: Experimental Design, Data Analysis, and Probing Macromolecule/Ligand Binding and Kinetic Interactions. In Methods in Cell Biology; Academic Press, 2008; Vol. 84, pp 79-113. https://doi.org/https://doi.org/10.1016/S0091-679X(07)84004-0.

(9) Wang, Y.; Wang, G.; Moitessier, N.; Mittermaier, A. K. Enzyme Kinetics by Isothermal Titration Calorimetry: Allostery, Inhibition, and Dynamics. Frontiers in Molecular Biosciences. 2020, p 285.

(10) Zambelli, B. Characterization of Enzymatic Reactions Using ITC. In Microcalorimetry of Biological Molecules: Methods and Protocols; Ennifar, E., Ed.; Springer New York: New York, NY, 2019; pp 251-266. https://doi.org/10.1007/978-1-4939-9179-2_18.

(11) Olsson, T. S. G.; Williams, M. A.; Pitt, W. R.; Ladbury, J. E. The Thermodynamics of ProteinLigand Interaction and Solvation: Insights for Ligand Design. J. Mol. Biol. 2008, 384, 10021017. https://doi.org/10.1016/j.jmb.2008.09.073.

(12) Caro, J. A.; Harpole, K. W.; Kasinath, V.; Lim, J.; Granja, J.; Valentine, K. G.; Sharp, K. A.; Wand, A. J. Entropy in Molecular Recognition by Proteins. Proc. Natl. Acad. Sci. U. S. A. 2017, 114, 6563-6568. https://doi.org/10.1073/pnas.1621154114.

(13) Swainston, N.; Baici, A.; Bakker, B. M.; Cornish-Bowden, A.; Fitzpatrick, P. F.; Halling, P.; Leyh, T. S.; O’Donovan, C.; Raushel, F. M.; Reschel, U.; Rohwer, J. M.; Schnell, S.; Schomburg, D.; Tipton, K. F.; Tsai, M.-D.; Westerhoff, H. V; Wittig, U.; Wohlgemuth, R.; Kettner, C. STRENDA DB: Enabling the Validation and Sharing of Enzyme Kinetics Data. FEBS J. 2018, 285, 21932204. https://doi.org/https://doi.org/10.1111/febs.14427.

(14) Halling, P.; Fitzpatrick, P. F.; Raushel, F. M.; Rohwer, J.; Schnell, S.; Wittig, U.; Wohlgemuth, R.; Kettner, C. An Empirical Analysis of Enzyme Function Reporting for Experimental Reproducibility: Missing/Incomplete Information in Published Papers. Biophys. Chem. 2018, 
242, 22-27. https://doi.org/https://doi.org/10.1016/j.bpc.2018.08.004.

(15) Grosch, J.-H.; Sieben, M.; Lattermann, C.; Kauffmann, K.; Büchs, J.; Spieß, A. C. Enzyme Activity Deviates Due to Spatial and Temporal Temperature Profiles in Commercial Microtiter Plate Readers. Biotechnol. J. 2016, 11, 519-529. https://doi.org/https://doi.org/10.1002/biot.201500422.

(16) Grosch, J.-H.; Wagner, D.; Knaup, N.; Keil, T.; Spieß, A. C. Influence of the Experimental Setup on the Determination of Enzyme Kinetic Parameters. Biotechnol. Prog. 2017, 33, 87-95. https://doi.org/https://doi.org/10.1002/btpr.2390.

(17) Stroberg, W.; Schnell, S. On the Estimation Errors of KM and V from Time-Course Experiments Using the Michaelis-Menten Equation. Biophys. Chem. 2016, 219, 17-27. https://doi.org/https://doi.org/10.1016/j.bpc.2016.09.004.

(18) Quality control of recombinant protein: Best practice recommendations https://arbremobieu.eu/guidelines-on-protein-quality-control/ (accessed Mai 05, 2021).

(19) Baker, M. Is There a Reproducibility Crisis? Nature 2016, 533, 452-454. https://doi.org/10.1038/533452A.

(20) Lorber, B.; Fischer, F.; Bailly, M.; Roy, H.; Kern, D. Protein Analysis by Dynamic Light Scattering: Methods and Techniques for Students. Biochem. Mol. Biol. Educ. 2012, 40, 372-382. https://doi.org/https://doi.org/10.1002/bmb.20644.

(21) Salis, A.; Bilaničová, D.; Ninham, B. W.; Monduzzi, M. Hofmeister Effects in Enzymatic Activity: Weak and Strong Electrolyte Influences on the Activity of Candida Rugosa Lipase. J. Phys. Chem. B 2007, 111, 1149-1156. https://doi.org/10.1021/jp066346z.

(22) Goldberg, R. N.; Kishore, N.; Lennen, R. M. Thermodynamic Quantities for the lonization Reactions of Buffers. J. Phys. Chem. Ref. Data 2002, 31, 231-370. https://doi.org/10.1063/1.1416902.

(23) Henderson, K. L.; Francis, D. H.; Lewis, E. A.; Emerson, J. P. Thermodynamics of Substrate Binding to the Metal Site in Homoprotocatechuate 2,3-Dioxygenase: Using ITC under Anaerobic Conditions to Study Enzyme-Substrate Interactions. Biochim. Biophys. Acta - Gen. Subj. 2016, 1860, 910-916. https://doi.org/https://doi.org/10.1016/j.bbagen.2015.07.013.

(24) Viña-Gonzalez, J.; Alcalde, M. Directed Evolution of the Aryl-Alcohol Oxidase: Beyond the Lab Bench. Comput. Struct. Biotechnol. J. 2020, 18, 1800-1810. https://doi.org/https://doi.org/10.1016/j.csbj.2020.06.037.

(25) Ewing, T. A.; Gygli, G.; Fraaije, M. W.; van Berkel, W. J. H. Chapter Four - Vanillyl Alcohol Oxidase. In Flavin-Dependent Enzymes: Mechanisms, Structures and Applications; Chaiyen, P., Tamanoi, F. B. T.-T. E., Eds.; Academic Press, 2020; Vol. 47, pp 87-116. https://doi.org/https://doi.org/10.1016/bs.enz.2020.05.003.

(26) Toplak, M.; Matthews, A.; Teufel, R. The Devil Is in the Details: The Chemical Basis and Mechanistic Versatility of Flavoprotein Monooxygenases. Arch. Biochem. Biophys. 2021, 698, 108732. https://doi.org/https://doi.org/10.1016/j.abb.2020.108732.

(27) Müller, M.; Katzberg, M.; Bertau, M.; Hummel, W. Highly Efficient and Stereoselective Biosynthesis of $(2 \mathrm{~S}, 5 \mathrm{~S})$-Hexanediol with a Dehydrogenase from Saccharomyces Cerevisiae. Org. Biomol. Chem. 2010, 8, 1540-1550. https://doi.org/10.1039/B920869K.

(28) Bitterwolf, P.; Gallus, S.; Peschke, T.; Mittmann, E.; Oelschlaeger, C.; Willenbacher, N.; Rabe, K. S.; Niemeyer, C. M. Valency Engineering of Monomeric Enzymes for Self-Assembling Biocatalytic Hydrogels. Chem. Sci. 2019, 10, 9752-9757. 
https://doi.org/10.1039/C9SC04074A.

(29) Guo, P.-C.; Bao, Z.-Z.; Ma, X.-X.; Xia, Q.; Li, W.-F. Structural Insights into the Cofactor-Assisted Substrate Recognition of Yeast Methylglyoxal/Isovaleraldehyde Reductase Gre2. Biochim.

Biophys. Acta - Proteins Proteomics 2014, 1844, 1486-1492.

https://doi.org/https://doi.org/10.1016/j.bbapap.2014.05.008.

(30) Gallus, S.; Peschke, T.; Paulsen, M.; Burgahn, T.; Niemeyer, C. M.; Rabe, K. S. Surface Display of Complex Enzymes by in Situ SpyCatcher-SpyTag Interaction. ChemBioChem 2020, 21, 21262131. https://doi.org/https://doi.org/10.1002/cbic.202000102.

(31) Skoupi, M.; Vaxelaire, C.; Strohmann, C.; Christmann, M.; Niemeyer, C. M. EnantiogroupDifferentiating Biocatalytic Reductions of Prochiral Cs-Symmetrical Dicarbonyl Compounds to Meso Compounds. Chem. - A Eur. J. 2015, 21, 8701-8705. https://doi.org/https://doi.org/10.1002/chem.201500741.

(32) Burgahn, T.; Pietrek, P.; Dittmeyer, R.; Rabe, K. S.; Niemeyer, C. M. Evaluation of a Microreactor for Flow Biocatalysis by Combined Theory and Experiment. ChemCatChem 2020, 12, 2452-2460. https://doi.org/10.1002/cctc.202000145.

(33) Peschke, T.; Bitterwolf, P.; Gallus, S.; Hu, Y.; Oelschlaeger, C.; Willenbacher, N.; Rabe, K. S.; Niemeyer, C. M. Self-Assembling All-Enzyme Hydrogels for Flow Biocatalysis. Angew. Chemie Int. Ed. 2018, 57, 17028-17032. https://doi.org/10.1002/anie.201810331.

(34) Li, H.; Forson, B.; Eckshtain-Levi, M.; Valentino, H.; Martín del Campo, J. S.; Tanner, J. J.; Sobrado, P. Biochemical Characterization of the Two-Component Flavin-Dependent Monooxygenase Involved in Valanimycin Biosynthesis. Biochemistry 2021, 60, 31-40. https://doi.org/10.1021/acs.biochem.0c00679.

(35) Lee, C. W.; Park, S.-H.; Lee, S. G.; Park, H. H.; Kim, H. J.; Park, H.; Park, H.; Lee, J. H. Crystal Structure of Dihydrodipicolinate Reductase (PaDHDPR) from Paenisporosarcina Sp. TG-14: Structural Basis for NADPH Preference as a Cofactor. Sci. Rep. 2018, 8, 7936. https://doi.org/10.1038/s41598-018-26291-x.

(36) Martinelli, L. K. B.; Ducati, R. G.; Rosado, L. A.; Breda, A.; Selbach, B. P.; Santos, D. S.; Basso, L. A. Recombinant Escherichia Coli GMP Reductase: Kinetic, Catalytic and Chemical Mechanisms, and Thermodynamics of Enzyme-Ligand Binary Complex Formation. Mol. Biosyst. 2011, 7, 1289-1305. https://doi.org/10.1039/COMB00245C.

(37) Breicha, K.; Müller, M.; Hummel, W.; Niefind, K. Crystallization and Preliminary Crystallographic Analysis of Gre2p, an NADP+-Dependent Alcohol Dehydrogenase from Saccharomyces Cerevisiae. Acta Crystallogr. Sect. F Struct. Biol. Cryst. Commun. 2010, 66, 838-841. https://doi.org/10.1107/S1744309110018889.

(38) Hydrodynamic Radius Converter https://www.fluidic.com/resources/Toolkit/hydrodynamicradius-Converter/ (accessed Mai 05, 2021).

(39) Inoue, Y.; Rhee, H.; Watanabe, K.; Murata, K.; Kimura, A. Metabolism of 2-oxoaldehyde in Mold: Purification and Characterization of Two Methylglyoxal Reductases from Aspergillus Niger. Eur. J. Biochem. 1988, 171, 213-218. https://doi.org/10.1111/j.14321033.1988.tb13778.x.

(40) Murata, K.; Fukuda, Y. Metabolism of 2-oxoaldehyde in Yeasts: Purification and Characterization of NADPH-Dependent Methylglyoxal-Reducing Enzyme from Saccharomyces Cerevisiae. Eur. J. Biochem. 1985, 151, 631-636. https://doi.org/10.1111/j.14321033.1985.tb09151.x

(41) Akita, H.; Watanabe, M.; Suzuki, T.; Nakashima, N.; Hoshino, T. Molecular Cloning and 
Characterization of Two YGL039w Genes Encoding Broad Specificity NADPH-Dependent Aldehyde Reductases from Kluyveromyces Marxianus Strain DMB1. FEMS Microbiol. Lett. 2015, 362, 1-6. https://doi.org/10.1093/femsle/fnv116.

(42) Inoue, Y.; Tran, L.-T.; Yoshikawa, K.; Murata, K.; Kimura, A. Purification and Characterization of Glyoxalase I from Hansenula Mrakii. J. Ferment. Bioeng. 1991, 71, 131-133. https://doi.org/10.1016/0922-338X(91)90239-D.

(43) Wiseman, T.; Williston, S.; Brandts, J. F.; Lin, L. N. Rapid Measurement of Binding Constants and Heats of Binding Using a New Titration Calorimeter. Anal. Biochem. 1989, 179, 131-137. https://doi.org/10.1016/0003-2697(89)90213-3.

(44) Malvern Instruments Ltd. MICROCAL PEAQ-ITC User Manual. 2015.

(45) Maruno, T.; Ohkubo, T.; Uchiyama, S. Stirring Rate Affects Thermodynamics and Unfolding Kinetics in Isothermal Titration Calorimetry. J. Biochem. 2020, 168, 53-62. https://doi.org/10.1093/jb/mvaa028.

(46) Baici, A. Kinetics of Enzyme-Modifier Interactions; $1^{\text {st }}$ ed.; Springer, Vienna, 2015. https://doi.org/10.1007/978-3-7091-1402-5.

(47) Bauduin, P.; Nohmie, F.; Touraud, D.; Neueder, R.; Kunz, W.; Ninham, B. W. Hofmeister Specific-lon Effects on Enzyme Activity and Buffer PH: Horseradish Peroxidase in Citrate Buffer. J. Mol. Liq. 2006, 123, 14-19. https://doi.org/10.1016/j.molliq.2005.03.003.

(48) Hollmann, S.; Frohme, M.; Endrullat, C.; Kremer, A.; D’Elia, D.; Regierer, B.; Nechyporenko, A. Ten Simple Rules on How to Write a Standard Operating Procedure. PLoS Comput. Biol. 2020, 16, 1-10. https://doi.org/10.1371/journal.pcbi.1008095.

(49) STRENDA Guidelines https://www.beilstein-institut.de/en/projects/strenda/guidelines/ (accessed Mai 05, 2021).

(50) Wolstencroft, K.; Krebs, O.; Snoep, J. L.; Stanford, N. J.; Bacall, F.; Golebiewski, M.; Kuzyakiv, R.; Nguyen, Q.; Owen, S.; Soiland-Reyes, S.; Straszewski, J.; van Niekerk, D. D.; Williams, A. R.; Malmström, L.; Rinn, B.; Müller, W.; Goble, C. FAIRDOMHub: A Repository and Collaboration Environment for Sharing Systems Biology Research. Nucleic Acids Res. 2017, 45, D404-D407. https://doi.org/10.1093/nar/gkw1032.

(51) NFDI4Chem https://nfdi4chem.de/ (accessed Mai 05, 2021).

(52) NFDI4Cat http://gecats.org/NFDI4Cat.html (accessed Mai 05, 2021).

(53) NFDI4Ing https://nfdi4ing.de/ (accessed Mai 05, 2021).

(54) GO FAIR Initiative https://www.go-fair.org/go-fair-initiative/ (accessed Mai 05, 2021).

(55) Bommarius, A. S.; Paye, M. F. Stabilizing Biocatalysts. Chem. Soc. Rev. 2013, 42, 6534-6565. https://doi.org/10.1039/c3cs60137d.

(56) Sheldon, R. A.; Woodley, J. M. Role of Biocatalysis in Sustainable Chemistry. Chem. Rev. 2018, 118, 801-838. https://doi.org/10.1021/acs.chemrev.7b00203.

(57) Žoldák, G.; Sprinzl, M.; Sedlák, E. Modulation of Activity of NADH Oxidase from Thermus Thermophilus through Change in Flexibility in the Enzyme Active Site Induced by Hofmeister Series Anions. Eur. J. Biochem. 2004, 271, 48-57. https://doi.org/10.1046/j.14321033.2003.03900.x.

(58) Bauduin, P.; Renoncourt, A.; Touraud, D.; Kunz, W.; Ninham, B. W. Hofmeister Effect on Enzymatic Catalysis and Colloidal Structures. Curr. Opin. Colloid Interface Sci. 2004, 9, 43-47. 
https://doi.org/10.1016/j.cocis.2004.05.031.

(59) Chen, C.-L.; Paul, L. N.; Mermoud, J. C.; Steussy, C. N.; Stauffacher, C. V. Visualizing the Enzyme Mechanism of Mevalonate Diphosphate Decarboxylase. Nat. Commun. 2020, 11, 3969. https://doi.org/10.1038/s41467-020-17733-0.

(60) Lu, C.; Peng, X.; Lu, D.; Liu, Z. Global and Kinetic Profiles of Substrate Diffusion in Candida Antarctica Lipase B: Molecular Dynamics with the Markov-State Model. ACS Omega 2020, 5, 9806-9812. https://doi.org/10.1021/acsomega.9b04432.

(61) Kokkonen, P.; Beier, A.; Mazurenko, S.; Damborsky, J.; Bednar, D.; Prokop, Z. Substrate Inhibition by the Blockage of Product Release and Its Control by Tunnel Engineering. RSC Chem. Biol. 2021. https://doi.org/10.1039/d0cb00171f.

(62) Gygli, G.; Lucas, M. F.; Guallar, V.; van Berkel, W. J. H. The Ins and Outs of Vanillyl Alcohol Oxidase: Identification of Ligand Migration Paths. PLoS Comput. Biol. 2017, 13, 1-27. https://doi.org/10.1371/journal.pcbi.1005787.

(63) Estévez-Gay, M.; Iglesias-Fernández, J.; Osuna, S. Conformational Landscapes of Halohydrin Dehalogenases and Their Accessible Active Site Tunnels. Catalysts 2020, 10, 1-14. https://doi.org/10.3390/catal10121403.

(64) Siedhoff, N. E.; Schwaneberg, U.; Davari, M. D. Chapter Twelve - Machine Learning-Assisted Enzyme Engineering. In Enzyme Engineering and Evolution: General Methods; Tawfik, D. S. B. T.-M. in E., Ed.; Academic Press, 2020; Vol. 643, pp 281-315. https://doi.org/https://doi.org/10.1016/bs.mie.2020.05.005.

(65) Yang, K. K.; Wu, Z.; Arnold, F. H. Machine-Learning-Guided Directed Evolution for Protein Engineering. Nat. Methods 2019, 16, 687-694. https://doi.org/10.1038/s41592-019-0496-6.

(66) Mazurenko, S.; Prokop, Z.; Damborsky, J. Machine Learning in Enzyme Engineering. ACS Catal. 2020, 10, 1210-1223. https://doi.org/10.1021/acscatal.9b04321.

(67) Truppo, M. D. Biocatalysis in the Pharmaceutical Industry: The Need for Speed. ACS Med. Chem. Lett. 2017, 8, 476-480. https://doi.org/10.1021/acsmedchemlett.7b00114. 
For Table of Contents Only

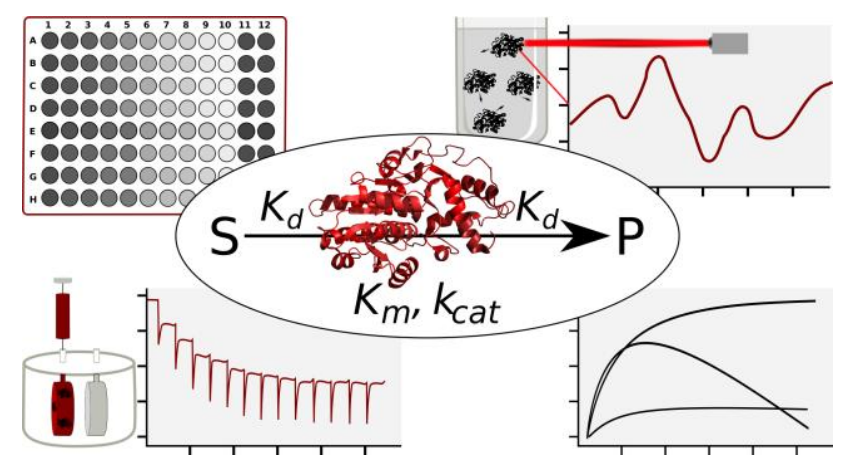

